\title{
Fifty Years On: Founding Psychoanalytic Psychotherapy ${ }^{\#}$
}

\author{
Michele Minolli ${ }^{\dagger *}$
}

ABSTRACT. - Fifty years after Freud's death we feel the time has come to revisit epistemic assumptions and Freudian theory. The Italian Society of Relationship Psychoanalysis (S.I.P.Re) is about to launch a new psychoanalytic journal, open to all, as a space for discussion, exchange, and research. It is generally known that Freud was the greatest contributor to the study of psychic distress in the nineteenth and twentieth centuries. It is appropriate to look to Freud, then, for the theoretical and technical basis for psychoanalytic psychotherapy with the aim of making psychoanalysis practicable for neuroses and psychoses, in the private and public sectors. Our study and research efforts are channeled towards Ricerca Psicoanalitica as the tangible result of the project.

Writing the editorial of a new journal should be relatively simple. There have been numerous discussions, clarifications, and explanations in countless design meetings, such that a summary would perhaps suffice. That is not the case.

The richness of creative intuition is not easily translated into words. Addressing this collective need for a printed context for thoughts and ideas in the field of psychoanalysis is not something automatic to be taken for granted.

A well-formulated presentation is therefore necessary.

The title itself, Ricerca Psicoanalitica, defines the pre-set objective of the journal: psychoanalysis and research.

Periodically, the death knell announcing the end of psychoanalysis is to be heard, but we believe that Freudian theory represents the most complete, logical, and articulate theoretical construct by which we explain and understand mental illness that the last two centuries have produced.

That is not to say, however, that Freudian theory cannot or should not be examined and revisited critically and with intellectual honesty. Research, in

${ }^{\#}$ Ricerca Psicoanalitica, 1990, Year I, n. 1, pp. 5-12.

*(1935-2020). SIPRe - Società Italiana di Psicoanalisi della Relazione, Past President and Past Editor in Chief. 
fact, is a constructive attitude, dependent upon the conviction that every theory is inevitably subject to evolution-revolution (Khun).

Although excellent national and international psychoanalysis journals already exist, they tend to be the official expression of the various psychoanalytic societies. Any institution, regardless of its structure, intent and purpose, tends to safeguard itself, to keep faith with its origins, and maintain the status quo.

Leafing through existing psychoanalysis journals, at the risk of undue generalization, it is striking that, Freudian thought is increasingly taken for granted, established, and untouchable, and focuses almost exclusively on recent authors who fail to make historical and theoretical connections.

Thus, fifty years after Freud's death we think the time has come to take an un-institutionalized approach to Freud's work.

A real, concrete opportunity to overcome the crystallized vision of psychoanalysis will allow us to assume with clarity, the position of someone who is not simply limited to a consideration of partial or marginal aspects with a view to developing or clarifying them: our theoretical space leads us to consider the Freudian construct in its historical entirety without prejudice and above all without epistemic a priori reasoning.

A journal has a momentum of its own that takes it to places that its authors cannot foresee.

It is legitimate and useful, however, to be explicit about the foreseeable direction and objectives of the journal.

Ricerca Psicoanalitica may be distinguished in its choices of historical significance: clinical applicability which is totally dependent on appropriate theorizing, and method.

Freud was certainly a milestone in the history of humanity. His merit lay in expanding the horizons of human beings past the limits of consciousness into the unconscious territories of hic sunt leones. Freud had the courage to attempt to build a theory on the workings of the psyche by demythologizing the prevailing anthropocentric vision. Lastly, it was Freud who shouldered the burden of daring to pronounce on mental illness and implement interventions in the search for its meaning, and in the attempt to make sense of it.

Notwithstanding Freud's genius, as with all scientific theories, we must choose the level at which to set the problem as well as the theoretical efforts to explain and demonstrate it. The problem, today, as in the past, is mental illness. While Janet explored degeneration; Charcot, heredity; Brucke, the nervous system; and Breuer, hypnoid states; Freud chose to explain mental illness from the inside as integral to the specific functioning of the human being.

While we realize that this level, albeit unexplored and unfulfilled, retains its genius and relevance, we believe that its theoretical construct requires thorough and ample reconsideration. 
In Italy, there is growing awareness of the epistemic and theoretical grounds for this conviction. Anachronistically, describing them here would be previewing specific content. We can, however, indicate some grounds of a practical and clinical nature which reveal the overlooked operational level of psychoanalysis.

Historically, psychoanalysis has had some difficulty in establishing itself, or rather, has failed to establish itself in public institutions for the treatment of mental illness.

Apart from some well-known institutions and some sporadic isolated instances, the inclusion of psychoanalytic therapy in the public sector is either considered impossible a priori, or considered ineffectual because inapplicable.

There is also the futility of the psychoanalytic method with regard to psychosis.

We refer, here, not so much to Freudian statements, but rather to the distortion of the method as it has been applied to 'narcissistic neuroses'.

These reasons are not as fortuitous and superficial as they might seem, in fact, they are closely connected and dependent on a set of global problems that psychoanalysis has increasingly, and very noticeably, addressed since the New York Congress, in 1958.

In fact, if a theory which presents itself as a general theory of mental illness fails to assume importance in public interventions and fails to deal effectively with psychosis, it clearly requires rethinking and revisiting (Scano).

Thus, Ricerca Psicoanalitica aims to investigate a psychoanalytic theory which, free from the epistemic burdens of its historic formulation, while holding onto the central Freudian concept of the 'subjective', finds, or sets out to find, a general explanation of the ever-present problem of 'mental illness'. An appropriate formulation would guarantee interventions on neurosis and psychosis both in the private and public sectors.

In recent times, the distinction between psychoanalysis and psychoanalytic psychotherapy has acquired more substance in the psychoanalytic environment. One definition, which takes account of reality, identifies 'psychoanalysis' with Freudian thought and method, as the Gesammelte Werke have historically been represented; it uses the term 'psychoanalytic psychotherapy' for formulations that differ while remaining psychoanalytic.

Ricerca Psicoanalitica, in adopting this terminological distinction, functions as a psychoanalytic psychotherapy journal insofar as we believe we need to go beyond Freudian orthodoxy and move towards theoreticalmethodological principles which make explicit references to valid but nonstandard interpretations.

The second choice we need to clarify is method. Freud believed his statements were deduced exclusively from the observation of patients, and, while leaving room for perfectibility in subsequent research and verifica- 
tion, observed data has remained dependent on the psychoanalytic method.

With Popper, the rigidity of this position no longer has much scientific citizenship: epistemic a priori reasoning and personal Weltanschauung have been exposed as inevitably conditioning theoretical constructs.

And yet, regardless of the issues related to validation, inference remains the path to developing theory.

Today, it may be possible to see things more clearly. The observation of clinical data has always led to the construction of clinical theory: a theory that attempts to explain intrapsychic and subjective data. However, clinical data is already the result of the intrapsychic and the subjective, not only of the patient, but also of the psychoanalyst.

We believe that we cannot be content with 'narrative truth' as it not only fails to provide a thorough explanation, but refers back theoretically to the search for an explanation based on 'historical truth'.

An analysis of phenomenic data subject to experimental observation may fail to lead to clinical theory, but may produce extremely useful material for the verification and validation-falsification of some basic assumptions.

For example, the enormous amount of material brought to light in recent years by evolutionary experimental psychology might have prevented Freud from conceptualizing primary narcissism and Malher from building a theory on original indifferentiation.

Since exponents of psychoanalysis set up the procedure of experimental observation, beginning with Spitz and leading to Stern, a vein referred to as 'experimental psychoanalysis' came into being which is methodologically close to the procedure for experimental developmental psychology.

Today, these positions constitute both a challenge to psychoanalysis and a stimulus for critical revisionism. A consideration of these new experimental contributions necessarily leads to a revisitation of the basic theoretical concepts of psychoanalysis (Dazzi).

Ricerca Psicoanalitica intends to adopt a bridging method that correlates the observation of intra-setting clinical data with the observation of experimental data. Keeping the two methods in mind will be neither easy nor restful, but the feat will be worth attempting to develop a new formulation of the 'theory of the psychological subject', which, from within the historical groove of Freudian tradition will renew 'outdated' epistemic assumptions, and introduce empirically sound methods of verification with the exactness and rigor that modern day research ensures.

Keeping in mind the conjugality of empirical and theoretical data, as well as giving space to topics arising from experimental observation at the articles' very inception should not only be possible but also extremely stimulating.

The concreteness of the project is assured by describing the programmatic areas that, at least in this period, seem to us to be priorities. 
The issues themselves will afford the reader the opportunity to validate their usefulness in theoretical terms, but perhaps more importantly, in explanatory terms.

Separating areas of research is hardly legitimate since they are so closely connected and interdependent, but to give you an overview it is useful to list the areas of research in detail:

The Epistemic area, that has two moments

- an explanation of the scientific-cultural relationships underlying Freudian theorization in a historical perspective;

- research of alternative epistemic references for a theoretical model in line with the premises of current philosophy of science.

The Theoretical area, it addresses various and interrelated issues:

- consideration that the formulation of metapsychology is variable, depending on the epistemic assumptions characteristic of the Central European scientific environment at the turn of the century;

- verification of the grade of internal coherence of Freudian construct, consequently highlighting the inevitable aporias;

- the need to achieve a level of unity between 'theoretical' theory and clinical theory in order to overcome the current dichotomy inherent in the coexistence of two epistemically irreconcilable and ideologically contrasting theories;

- the development, within a critical-revisionist work, of theoretical assumptions accountable both to formalization and verification.

Scientific Methodology: the general relationship between method and science.

Today, this is a priority consideration: because of inevitable epistemic revision, the scientific nature of the method cannot be taken for granted. Method and theory are so closely related that it is useful always to consider this interdependence in order to avoid absolutizing theoretical statements that are part of the model-building process.

Specific Psychoanalysis Methodology: the general connection between psychoanalytic methodology and scientific methodology in general. Psychoanalysis, to be science, must establish its own specific method within the broader scientific method. Specific psychoanalytic methodology must be reconsidered both in light of the current episteme and in terms of its applicability.

The Clinical area: it concerns what today we call the general theory of procedure.

Further study should ultimately lead to directing the concepts of procedural theory or clinical theory as much towards general theory as towards method.

In this regard, the existence in the Freudian corpus of 'clinical theory' alongside 'theoretical theory' seems to be the clearest sign of malaise and 
inconsistency. The concepts of clinical theory have a certain power of suggestion in relation to their high degree of concreteness; it is feasible, therefore, that an in-depth analysis constitutes a possible route to reaching a single formulation of 'General Theory'.

The other aspect of the clinical context is verifiability. Both the public situation, individual intervention, and group therapy can and should be carefully considered as natural settings for verification. The contribution of these three operational settings thus constitute a valuable and indispensable contribution for reflection and theorization.

We do not consider this task easy, but we are convinced that a clearly defined research programme in this direction can be a stimulus for convergence of study and research.

Ricerca Psicoanalitica is an initiative of the Italian Society of Relationship Psychoanalysis (S.I.P.Re.).

The journal is conceived as an open space for dialogue and discussion on the problematic nature of psychoanalysis, as well as a response to the need for expression and stimulation of theoretical-clinical research at S.I.P.Re.

It is right, therefore, to present schematically, the conceptual framework within which S.I.P.Re moves.

In explaining human behavior, we consider it fundamental to adopt a unitary and unifying concept such as that of the ego as subject and identity in line with the Freudian idea of 'Project' (1895), a work in which the ego, aside from its neurological code, presents itself as an organization.

Subjectivity and identity seem to be two moments based respectively on simple consciousness and on self-consciousness (Jervis), which determine the formation of individuality.

Without negating the biological components of the human organism rendered absolute by Freudian drive, we believe that the structuring of the ego as subject and as identity takes place within the relationship.

In this perspective, the theoretical reflection advanced by so-called experimental psychoanalysis (Stern) is inspiring.

Note that we do not use the term 'relationship' in the sense of the interpersonal relationship of the American culturalist approach (Sullivan, Horney, Fromm), nor is its meaning linked to 'object relations theory'. The latter, in its historical differentiations, led to the formation of a concept of 'relationship' with a focus on the external real object, which determines the constitution of the internal object (Fairbairn, Guntrip, in indirect relationship with Ferenczi, Hermann, Balint, Spitz and in a certain sense with Bowlby and Winnicott), or as a consequence of internal objects dependent on innate ghosts (M.Klein, Rosenfeld, Meltzer, Bion); or as an expression of autonomous parts of the ego, albeit physiological, in their adaptation process (Hartmann, Malher, Jacobson, Kernberg and in a certain sense Kohut and Sandler). 
Finally, we believe that 'relationship' fails to assume even the phenomenic group meaning of the relational model of Palo Alto (Bateson, Watzlawick, Jackon, Haley).

First of all, we assign to 'relationship' a purely theoretical meaning: the 'mutual conditioning' between organism and object, an area for the creation of the structures and organization of subjectivity in the first instance, and then of identity.

'Relationship' then refers back to a 'diagnostic' meaning in reference to the modalities, the traits, the patterns, i.e., the structures, as the historical result of mutual conditioning, both at a conscious and unconscious level of each individual.

Finally, 'relationship' has methodological significance in so far that it is subject to observation, in particular with regard to the analytical relationship, and the reproduction of intrapsychic relational solutions managed, through the interplay of the opposites, absolutization-negation (Matte Blanco) according to the workings of the unconscious,

This summary of the basic hypotheses that S.I.P.Re. has formulated does not, perhaps, demonstrate the extent to which our referent is both psychoanalysis and Freudian psychoanalysis.

Beyond its theoretical construct, the level of approach chosen by Freud still seems, to us, to constitute the most appropriate and pertinent level.

In our view, Freud necessarily constitutes the starting point for understanding and formulating an explanation of the workings of the psyche and the cause of mental discomfort. 
